\title{
Evidence of a segregation ratio distortion of SMN1 alleles in spinal muscular atrophy
}

\author{
Laura Alias $^{1,2}$, Maria J Barceló ${ }^{1,2}$, Ignasi Gich ${ }^{3}$, Marta Estapé $^{1,2,}$, Juan Parra ${ }^{4}$, \\ Maria Amenedo ${ }^{4}$, Montserrat Baiget ${ }^{1,2}$ and Eduardo F Tizzano*,1,2
}

${ }^{1}$ Department of Genetics, Hospital de Sant Pau, Barcelona, Spain; ${ }^{2}$ Centre for Biomedical Research on Rare Diseases, Barcelona, Spain; ${ }^{3}$ Department of Epidemiology, Hospital de Sant Pau, Barcelona, Spain; ${ }^{4}$ Department of Obstetrics and Gynaecology Hospital de Sant Pau, Barcelona, Spain

Spinal muscular atrophy (SMA) is an autosomal recessive disorder characterised by degeneration and loss of the motor neurons of the anterior horn of the spinal cord. The absence of SMN1 is determinant to have SMA and parents of SMA patients are regarded as carriers of the disease. We compared the segregation ratio of the mutated allele and the wild-type allele of all the confirmed carrier parents assuming Mendelian proportions. Results of transmissions in $\mathbf{2 3 5}$ prenatal tests and in $\mathbf{1 2 8}$ unaffected siblings showed a statistically significant deviation in favour of the wild-type SMN1 allele. The number of affected foetuses and carriers were lower than that expected. No significant differences in the sex ratio or in the progenitor origin of the transmitted allele to the carriers were found. One hypothesis that has been advanced to account for the distortion observed in affected foetuses is the negative postzygote selection due to early miscarriage. However, given that the number of carriers in our series was lower than expected, prezygote events such as meiotic drive, survival of gametes or preferential fertilisation should also be considered. European Journal of Human Genetics (2007) 15, 1090-1093; doi:10.1038/sj.ejhg.5201886; published online 11 July 2007

Keywords: SMA; SMN gene; segregation ratio; carrier analysis

\section{Introduction}

Spinal muscular atrophy (SMA) is an autosomal recessive disorder resulting in the loss of motor neurons. SMA is classified by age of onset and motor milestones achieved in types I, II and III (severe, intermediate and mild form, respectively). Approximately $90 \%$ of patients suffering from the different forms of SMA lack both copies of the SMN1 gene. Although the SMN2 gene is the homologous SMN1 copy present in all patients, it is unable to prevent the disease. The SMN1 transcript is mainly full-length codifying to a complete protein, whereas a substantial

*Correspondence: Dr EF Tizzano, Department of Genetics, Genetics and Research Institute, Hospital de Sant Pau, Av. Padre Claret 167, 08025, Barcelona, Spain. Tel: 3493291 9361; Fax: 3493291 9494;

E-mail: etizzano@santpau.es

In memoriam.

Received 16 March 2007; revised 14 May 2007; accepted 6 June 2007; published online 11 July 2007 proportion of the SMN2 transcript lacks exon 7. Therefore, SMA patients generate low levels of SMN protein from the SMN2 gene. ${ }^{1,2}$

Parents of an SMA patient are regarded as carriers although de novo mutations or germinal mosaicisms have been described. ${ }^{3}$ The original segregation analyses in SMA have been used to determine the genetic nature and inheritance of the disease. ${ }^{4,5}$ After the identification of the SMA gene, molecular analysis has provided accurate patient and carrier diagnoses. Assuming Mendelian inheritance, it is also possible to ascertain whether each allele is represented equally in the gametes. Transmission ratio distortion (TRD) is a significant departure from Mendelian segregation and the evidence in humans is limited, for example it has been excluded in cystic fibrosis. ${ }^{6}$ However, TRD of the SMN1 allele in SMA has been reported. ${ }^{7}$ We analysed the segregation ratio of the SMN1 gene in the offspring of SMA parents in whom de novo mutations or 
germinal mosaicisms were ruled out. The results from prenatal analyses and from carrier studies in unaffected siblings are presented.

\section{Patients, materials and methods Patients}

A total of 235 prenatal tests were undertaken in 180 couples, who were proven carriers of SMA with at least one affected child (with SMN1 mutation) and one SMN1 copy in the quantitative DNA analysis. Furthermore, 128 offspring of these couples were analysed for carrier diagnosis. The SMA clinical type of the probandus was known for each family.

\section{SMA locus analysis}

All the couples, offspring and pregnancies were tested for deletions, SMN1 copy number and for C272(Ag1-CA) and C212 marker analysis as reported. ${ }^{8}$ Furthermore, these markers determined the maternal or paternal origin of the at-risk haplotype.

\section{Segregation analysis and statistics}

We compared the transmission ratio of the mutated allele and the wild-type allele of all the carrier parents, assuming an equal (1:1) distribution of both alleles. For prenatal tests, the expected frequencies were 25\% (homozygous affected; $-/-$ ), 50\% (heterozygous; $-/+$ ) and 25\% (noncarrier or wild type; $+/+)$ according to Mendelian inheritance. For unaffected siblings, the expected frequencies were 33\% (non-carrier) and 66\% (carrier). Furthermore, the paternal origin of the transmitted mutation and the gender of foetuses and offspring were established. Finally, the clinical status of the probandus as acute (type I) or chronic disease (types II and III) was considered. Analysis of $\chi^{2}$ was undertaken with SPSS14.0.

\section{Results}

Out of 470 transmissions of the SMN1 gene (235 prenatal testings), 270 were of the wild-type allele and 200 of the mutated allele $(P=0.001)$. As regards the Mendelian proportions, 49 foetuses (21\%) were homozygously deleted (expected $n=58.75), 102$ (43\%) were carriers (expected $n=117.5)$ and $84(36 \%)$ were homozygous for the wildtype allele (expected $n=58.75$ ). The transmission rate of the deleted gene copy was lower than expected $(P=0.001)$ (Figure 1, panel a). Furthermore, of the 128 unaffected siblings of patients, $74(58 \%)$ were carriers (expected $n=85)$ and $54(42 \%)$ were non-carriers (expected $n=43$; $P=0.039$ ) (Figure 1, panel b). Considering the unaffected foetuses and the siblings $(n=314)$, the number of carriers was $176(56 \%$, expected $n=209)$ and that of non-carriers was 138 (44\%, expected $n=105 ; P<0.0001)$.

Dividing the groups by gender (Table 1), TRD was maintained. When considering prenatal carriers and noncarriers with siblings, females $(n=151)$ were $58 \%$ carriers $(n=88)$ vs $42 \%$ non-carriers $(n=63 ; P=0.031)$, whereas males $(n=163)$ were $54 \%$ carriers $(n=88)$ vs $46 \%$ noncarriers $(n=75 ; P=0.001)$. Furthermore, no significant differences in the sex ratio of the foetuses and siblings were observed. Nor were there any differences in the paternal or maternal origin of the transmitted allele to the carriers. We observed 166 transmissions of the mutated allele to carriers of which 77 were of maternal origin and 89 of paternal origin $(P=0.337)$.

As for the SMA type of foetuses (Table 2, Prenatal), the distribution in type I $(n=183)$ showed the expected number of affected cases, but a decrease in carriers and an increase in non-carriers. In chronic types II and III cases $(n=52)$, the number of carriers resembled the expected ones. However, there was a significant decrease in the number of affected cases and an increase in non-carriers. Regarding the siblings, 62 belonged to a family with a type I probandus, whereas 66 had types II and III affected
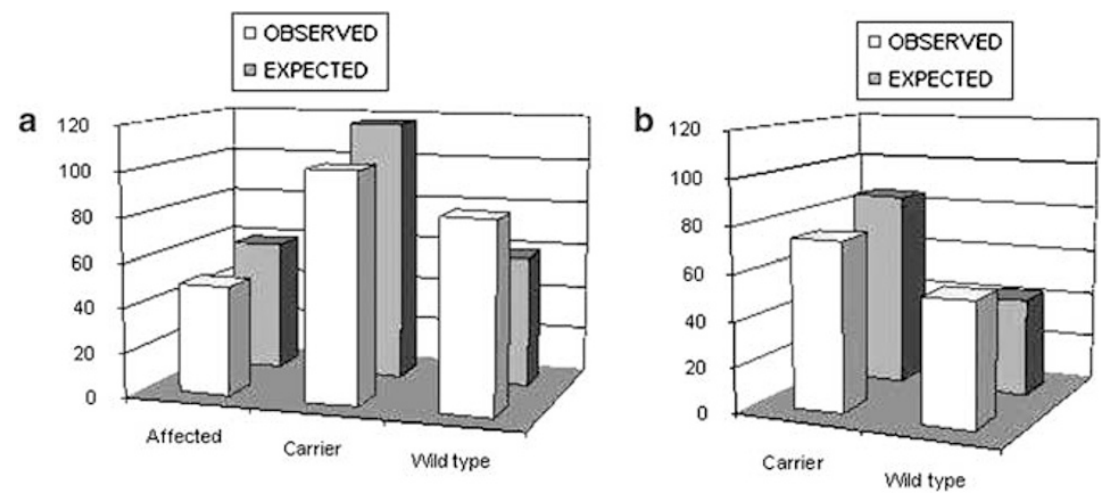

Figure 1 (a) Segregation ratio of SMN1 alleles in couples of carriers who requested prenatal diagnosis. The expected frequencies were 25, 50 and $25 \%$ assuming Mendelian inheritance. There are less affected carriers and more unaffected non-carriers (wild type). The transmission rate of the deleted gene copy was lower than expected $(P=0.001)$. (b) Segregation ratio of SMN1 alleles in siblings of SMA patients. The expected frequencies were $66 \%$ for carriers and $33 \%$ for non-carriers assuming Mendelian inheritance. The number of carriers was lower than expected $(P=0.039)$. 
Table 1 Results of the gender distribution in 235 prenatal diagnoses and 128 siblings of SMA patients according to the genotype

\begin{tabular}{lccccc}
\hline & \multicolumn{2}{c}{ Prenatal } & & \multicolumn{2}{c}{ Siblings } \\
\cline { 2 - 3 } \cline { 6 - 6 } Genotype & $X X$ & $X Y$ & & $X X$ & $X Y$ \\
\hline Affected (-/-) & $20(27.5)$ & $29(31.25)$ & & \\
Carrier (+/-) & $50(55)$ & $52(62.5)$ & & $38(41)$ & $36(45)$ \\
Wild type (+/+) & $40(27.5)$ & $44(31.25)$ & & $23(20)$ & $31(22)$ \\
Total & 110 & 125 & & 61 & 67 \\
Statistics $(P)$ & 0.016 & 0.028 & & 0.498 & 0.028 \\
\hline
\end{tabular}

Abbreviation: SMA, spinal muscular atrophy.

The expected numbers of Mendelian proportions are in brackets.

Table 2 Results of the clinical-type distribution in 235 prenatal diagnoses and 128 siblings of SMA patients according to the genotype

\begin{tabular}{|c|c|c|c|c|}
\hline \multirow[b]{2}{*}{ Genotype } & \multicolumn{2}{|c|}{ Prenatal } & \multicolumn{2}{|c|}{ Siblings } \\
\hline & Type I & $\begin{array}{l}\text { Types II } \\
\text { and III }\end{array}$ & Type I & $\begin{array}{l}\text { Types II } \\
\text { and III }\end{array}$ \\
\hline Affected (-/-) & $44(45.75)$ & $5(13)$ & & \\
\hline Carrier $(+/-)$ & $79(91.5)$ & $23(26)$ & $38(41)$ & $36(44)$ \\
\hline Wild type $(+/+)$ & $60(45.75)$ & $24(13)$ & $24(21)$ & $30(22)$ \\
\hline Total & 183 & 52 & 62 & 66 \\
\hline Statistics $(P)$ & 0.045 & 0.001 & 0.419 & 0.049 \\
\hline
\end{tabular}

Abbreviation: SMA, spinal muscular atrophy.

The expected numbers of Mendelian proportions are in brackets.

patient (Table 2, Siblings). However, the distribution between carriers and non-carriers in the type I cases (61 vs 39\%) was close to the expected (66.6 vs 33.3\%; $P=0.419$ ), whereas in types II and III families the distribution was almost equal (54.5 vs 45.5\%; $P=0.049$ ). Combining the SMA type in the probandus of the unaffected foetuses and the siblings (Table 2), the increase in the non-carrier individuals was also observed. Type I $(n=201)$ were $58 \%$ carriers $(n=117)$ vs $42 \%$ non-carriers ( $n=84 ; P=0.013)$, whereas types II and III $(n=113)$ were $52 \%$ carriers $(n=59) \quad$ vs $48 \%$ non-carriers $(n=54$; $P=0.001)$.

\section{Discussion}

This study describes the segregation of the SMN1 alleles in the offspring of a large number of proven SMA carrier couples based on prenatal samples and unaffected siblings. We investigated (1) the segregation of wild-type or mutated alleles according to the gender of the samples; (2) the paternal origin of the alleles; and (3) the distribution according to the SMA clinical type. Our results point to a possible distortion in the transmission ratio of the SMN1 alleles. This distortion appears to be independent of the progenitor that transmits the mutation and of the gender of the foetus or the offspring.
Before the availability of the SMN genetic test to confirm SMA disease, segregation ratios around 0.26 for type I SMA and 0.21 for chronic disease were described. ${ }^{4,5}$ These percentages did not differ considerably from the expected 0.25 as regards autosomal recessive inheritance. Moreover, a segregation analysis of 354 cases of chronic SMA pointed to a possible gender influence with a smaller number of females. ${ }^{9}$ Subsequently, by molecular analysis, a more reduced rate of SMA-affected pregnancies was noted, although these reports included couples without proven carrier status. ${ }^{10,11}$ A recent paper ${ }^{7}$ describes a TRD of the SMN1 allele based on the molecular analysis of 314 prenatal tests. These authors found a statistically significant deviation in favour of the wild-type allele (344 vs 284 $P=0.016)$ with a decrease in the number of affected foetuses $(20.7 \%)$. Interestingly, the observed frequencies of carriers (49\%) resembled the expected $50 \%$ and the frequency of non-carriers was increased $(30.3 \%){ }^{7}$ Combining prenatal data from this work with those of our series show a total of $614(344+270)$ transmissions of the wildtype allele and $484(284+200)$ of the mutated allele $(P<0.001)$. Furthermore, the proportion of affected foetuses is $20.7 \%$ (114/549, expected 137$), 46.6 \%$ of carriers (256, expected 275 ) and $32.6 \%$ of wild type (179, expected $137 ; P<0.001)$. These data provide further evidence that TRD is present in SMA.

Stratifying our data by clinical type, in type I cases we observed that the wild-type allele segregated more often than the mutated allele and that the number of carriers was lower than expected (Table 2). However, the number of affected foetuses was similar to that expected (44 vs 45.75). By contrast, in our types II and III cases, we observed less affected foetuses (5 observed vs 13 expected) with an increase in the wild-type cases (24 observed vs 13 expected). Other authors ${ }^{7}$ observed that the distortion was more marked in type I cases $(n=171)$ compared with types II and III cases $(n=48)$. However, these investigators included 35 additional samples with unknown clinical status, which could have influenced their observation.

The proportion of carriers in siblings of type I cases resembled that of expected, whereas the siblings of types II and III cases had a distortion of the $2 / 3$ (66\%) expected number of carriers. Indeed, the distribution in these siblings approached $50 \%$ (Table 2). These results suggest a tendency in the chronic families to preferentially transmit the wild-type allele.

One explanation proposed for the TRD in SMA is the negative postzygote selection owing to early miscarriage. ${ }^{7}$ However, our results indicating distortion also involved the carriers, who were not, a priori, prone to miscarriage. Thus, a second hypothesis is that prezygote events could also account for the TRD observed. Meiotic drive, survival of gametes or preferential fertilisation could be involved. ${ }^{12}$ SMN is an ubiquitously expressed protein and reduced levels of it causes SMA. ${ }^{1,2}$ It is not known whether lower 
SMN protein levels could result in a reduced fertilisation rate of a proportion of mutated gametes. Indeed, certain allele groups lacking SMN1 could be underrepresented, accounting for the distortion observed. However, chromosomes without SMN1 and SMN2 copies are present in humans and can be transmitted. ${ }^{13}$

In conclusion, we present evidence of a significant distortion in the segregation of SMN1 alleles in SMA families. This distortion is reflected in a smaller number of affected and carrier cases observed, and it appears more noticeable in chronic SMA chromosomes. A number of reasons may favour postzygote (that is, early miscarriage) or prezygote (that is, reduced fertilisation) explanations. However, both hypotheses are not mutually exclusive. To determine the significance of the segregation distortion in SMA biology and its consequences for genetic counselling, larger population studies in SMA families are warranted.

\section{Acknowledgements}

This work was supported by a grant from the Fondo de Investigación Sanitaria 05-2416. LA was supported by FUNDAME and CIBERER.

\section{References}

1 Coovert DD, Le TT, McAndrew PE et al: The survival motor neuron protein in spinal muscular atrophy. Hum Mol Genet 1997; 8: $1205-1214$
2 Lefebvre S, Burlet P, Liu Q et al: Correlation between severity and SMN protein level in spinal muscular atrophy. Nat Genet 1997; 16: 265-269.

3 Wirth B, Schmidt T, Hahnen E et al: De novo rearrangements found in $2 \%$ of index patients with spinal muscular atrophy: mutational mechanisms, parental origin, mutation rate, and implications for genetic counseling. Am J Hum Genet 1997; 61: $1102-1111$.

4 Pearn J: Genetic studies of acute infantile spinal muscular atrophy (SMA type I). J Med Genet 1978a; 15: 414-417.

5 Pearn J: Segregation analysis of chronic childhood spinal muscular atrophy. J Med Genet 1978b; 15: 418-423.

6 European Working Group on Cystic Fibrosis Genetics: No evidence for segregation distortion of cystic fibrosis alleles among sibs of cystic fibrosis patients. Eur J Hum Genet 1995; 3: 324-325.

7 Botta A, Tacconelli A, Bagni I et al: Transmission ratio distortion in the spinal muscular atrophy locus. Neurology 2005; 65: $1631-1635$

8 Barcelo MJ, Alias L, Caselles L, Robles Y, Baiget M, Tizzano E: Two independent mutations of the SMN1 gene in the same spinal muscular atrophy family branch: lessons for carrier diagnosis. Gen Med 2006; 8: 259-262.

9 Hausmanowa-Petrusewicz I, Zaremba J, Borkowska J, Szirkowiec W: Chronic proximal spinal muscular atrophy of childhood and adolescence: sex influence. J Med Genet 1984; 21: 447-450.

10 Ogino S, Leonard DG, Rennert H, Wilson RB: Spinal muscular atrophy genetic testing experience at an academic medical center. J Mol Diagn 2002; 4: 53-58.

11 Savas S, Eraslan S, Kantarci S et al: Prenatal prediction of childhood-onset spinal muscular atrophy (SMA) in Turkish families. Prenat Diagn 2002; 22: 703-709.

12 Lyttle T: Cheaters sometimes prosper: distortion of Mendelian segregation by meiotic drive. Trends Genet 1993; 9: 205-210.

13 Cuscó I, Barceló MJ, del Río E et al: Characterization of SMN hybrid genes in Spanish SMA patients: de novo, homozygous and compound heterozygous cases. Hum Genet 2001; 108: 222-229. 